\title{
BMJ Open Emergency Room Safer Transfer of Patients (ER-STOP): a quality improvement initiative at a community- based hospital to improve the safety of emergency room patient handovers
}

To cite: Norman S, DeCicco F, Sampson J, et al. Emergency Room Safer Transfer of Patients (ER-STOP): a quality improvement initiative at a community-based hospital to improve the safety of emergency room patient handovers. BMJ Open 2018;8:e019553. doi:10.1136/ bmjopen-2017-019553

- Prepublication history and additional material for this paper are available online. To view these files, please visit the journal online (http://dx.doi. org/10.1136/bmjopen-2017019553).

Received 11 September 2017 Revised 9 October 2018 Accepted 26 October 2018
Check for updates

(C) Author(s) (or their employer(s)) 2018. Re-use permitted under CC BY-NC. No commercial re-use. See rights and permissions. Published by BMJ.

For numbered affiliations see end of article.

Correspondence to

Savannah Norman;

savannahnorman@rcsi.com

\section{ABSTRACT}

Objectives Ensure early identification and timely management of patient deterioration as essential components of safe effective healthcare. Prompted by analyses of incident reports and deterioration events, a multicomponent organisational rescue from danger system was redesigned to decrease unexpected inpatient deterioration.

Design Quality improvement before-after unblinded trial. Setting 430-bed Canadian community teaching hospital. Participants All admitted adult medical-surgical patients in a before-after 12-month interventional study. Intervention Locally validated checklist (Modified Early Warning Score+urinary catheter in situ+nurse concern) with an intentional pause and explicit management options was deployed as a modification of an existing ward transfer of accountability fax report in the emergency department (ED).

Results Following deployment of Emergency Room Safer Transfer of Patients (ER-STOP), the risk of an unexpected CCRT (critical care response team) response within 24 hours of admission from ED to adult medical and surgical wards was significantly decreased $(0 \mathrm{R} 4.1,95 \% \mathrm{Cl}$ 2.17 to 7.77$)$. Mean $( \pm S D)$ ED wait times $(5.66 \pm 1.54 \mathrm{vs}$ $5.74 \pm 1.04$ hours, $p=0.30$ ), intensive care unit admission rate $(3.84 \%, n=233 v s 4.61 \%, n=278, p=0.06)$ and cardiac care unit admission rate $(9.51 \%, n=577 v s 9.60 \%, n=579$, $\mathrm{p}=0.198$ ) were unchanged.

Conclusions ER-STOP improvement was out of proportion to the predictive value of the checklist component suggesting that effectiveness of this lowcost sustainable tool was related to increased situational awareness, empowering a culture of patient safety and repurposing of an adjacent ED medical short-stay unit use. Local adaptation within existing processes is essential to successful safety outcomes.

\section{INTRODUCTION}

At our urban community teaching hospital, patient deterioration beyond the capacity of the inpatient ward care team is managed by a critical care response team (CCRT, locally known as Outreach) consisting of
Strengths and limitations of this study

- Intervention integrated into existing processes to ensure maximal sustainability.

- Promotional deployment and staff education in addition to top-down senior administrative support empowered staff to use Emergency Room Safer Transfer of Patients.

- Use of hybrid electronic health record system allowed for accurate and complete data sets to be used for analysis.

- Single-site deployment limits generalisability without local adaptation.

Limited by the before-and-after, unblinded design.

an intensivist, an intensive care unit (ICU) nurse and a respiratory therapist. Incident report analysis and an ongoing audit of CCRT responses identified opportunities for improvement in early identification and timely management of ward patient deterioration, including unanticipated deterioration within 24 hours of admission from the emergency department (ED). This prompted an organisational redesign of our rescue from danger system, with a targeted intervention to reduce the early, unexpected deterioration of patients admitted from the ED to our general medical and surgical wards.

Early identification and intervention of inpatient adult ward deterioration is an essential component of safe and effective healthcare. Critically ill patients admitted to a general ward that subsequently deteriorate and are transferred to a special care unit (eg, ICU, coronary care unit or high-dependency units) have increased morbidity and mortality in comparison with patients who are admitted directly from ED to a general ward. ${ }^{1}$ Validated scoring systems such as Modified Early Warning Score (MEWS) have been 
used to identify patients at risk of clinical deterioration. ${ }^{2}$ However, the predictive value of such tools remains uncertain largely due to methodological constraints. ${ }^{3}$ Additionally, the effectiveness of such scores appears to be context specific and dependent on a number of factors such as patient population and healthcare worker experience. ${ }^{4}$

Other tools to ensure patient safety in the acute care setting such as checklists have inconclusive clinical use. ${ }^{4}$ In practice, medical checklist adherence is often poor with variable levels of resistance to checklist implementation in acute care settings. ${ }^{5}$ Factors such as high reliance on verbal communication, ambiguity and poor staff communication have been previously identified as barriers to checklist use in acute care. ${ }^{6}$

Predictive limitations in early warning scores and feasibility constraints of implementing patient safety checklists or complex scoring in acute care settings highlight the currently limited options available to optimise patient safety during ED admissions. ${ }^{7}$ In practice, there is a large reliance on subjective clinical judgement, which inadequately prioritises patient safety. ${ }^{1}$

The aim of our study was to assess if implementation of a locally adapted checklist tool with an intentional pause and explicit management options would reduce by $50 \%$ unexpected CCRT responses to patient deterioration on general medical-surgical wards within 24 hours of admission from ED between January and December 2016 in comparison with the previous 12 months, without adversely affecting special care unit (SCU) use or ED waiting time for admission.

\section{METHODS}

Development of checklist-based intervention

The selection of ward medical-surgical ED admission population was identified based on review of severe adverse events and CCRT responses leading to SCU admission with specific exclusion of a special care (SCU), operating room, mental health, palliative or paediatric admissions.
In 2014, an exploratory study of locally available predictors for deterioration (1.6\% of ward patients) defined by CCRT response, mortality and SCU admission within 24 hours of ED admission (ie, vital signs, MEWS, $1 \mathrm{~L}$ or more intravenous fluids use, Foley catheter and non-invasive ventilation) was undertaken using matched (age, gender and admitting hospital unit, $n=19$ ) 2-month derivation and validation cohort (see Acknowledgement). Only two modestly predictive measures were identified: a MEWS greater than or equal to 3, and the presence of an indwelling Foley catheter (exceptions being pre-op or urological patients with acute urinary retention), the latter likely resulting from a previous evidence-based hospital-wide initiative to reduce Foley use. MEWS greater than 3 or Foley in situ had a $73.7 \%$ sensitivity (95\% CI $48.6 \%$ to $89.9 \%$ ) and a $78.9 \%$ specificity (95\% CI $53.9 \%$ to $93 \%$ ).

The checklist-based tool (MEWS+urinarycatheter in situ+nurseconcern) with an intentional pause and explicit management options within an existing ED-ward transfer of accountability fax report was co-designed by face-to-face consultation with ED staff and nursing leaders for optimal integration into existing ED care processes, ease of use and consensus agreement regarding the accuracy of terminology and clinical judgement for Foley catheter assessment (see figure 1).

If a patient is identified as high risk for deterioration, the admission is paused and the most responsible physician is contacted by the nursing staff to reassess the patient. Emergency Room Safer Transfer of Patients (ER-STOP) then prompts the physician to choose a number of specific ranked management options including admission to a SCU, scheduled follow-up with CCRT, admission to palliative end-of-life care or admission to the ward without increased monitoring.

\section{Data collection and measurement}

A single-centre unblinded before (1 January 2015 to 31 December 2015) and after (1 January 2016 to 31 December 2016) electronic health record 
(EHR) chart analysis of non-elective admissions to adult medical and surgical wards from the ED was performed. Patient demographics were assessed using a standardised national database resource intensity weighting based on case-mix grouping, age, comorbidity level, flagged interventions, intervention events and out-of-hospital interventions. ${ }^{8}$ The 1-year before-and-after time period was chosen to control for seasonal variation in admission volumes related to wintertime respiratory tract infection outbreaks and increased risk of SCU admission and ward deterioration related to surge conditions.

ER-STOP intervention performance was measured using unexpected adult inpatient ward deterioration occurring within 24 hours following ED admission requiring new unscheduled CCRT responses. Unexpected CCRT responses were defined as an unscheduled call to CCRT with a confirmed response documented in a team-completed audit form. The latter audit is part of an ongoing independent quality assurance intervention of CCRT responses that identifies CCRT improvement opportunities using secondary chart review by senior internists. Hospital-wide scheduled and unscheduled CCRT responses data were extracted from provincial critical care information system independently submitted from the investigators.

As a balancing measure, ED wait times (time from admitting order to arrival on ward) and SCU admission rates were measured to detect potentially adverse consequences of the intervention. Additionally, audit of ED admission documentation was done to further assess the impact and sustainability of our prediction tool. This was supplemented by qualitative information from verbal feedback from frontline ED staff actively using the tool and hospital administrative staff responses to deployment of the initiative. The proportion of pauses occurring each month in SCU admissions was monitored to assess process deployment and quantitate any ramp-up effect.

\section{Promotion and deployment}

The promotion of our tool in the ED consisted of small group education about the ER-STOP checklist and the effect on the admissions process. In addition, nursing practice leaders and supervisors attended daily unit huddles to discuss and encourage using ER-STOP as a patient safety initiative, with one-on-one education of the staff members in the Departments of Medicine and Surgery. The initiative was featured in the hospital-wide newsletter and hospital interdisciplinary quality improvement grand rounds. Lastly, the initiative was supported with the use of promotional posters within the ED and on huddle boards throughout the organisation. This occurred from September 2015 to December 2015.

Following institution-wide promotion and education, ER-STOP was deployed in January 2016. Nursing practice leaders and supervisors within the ED supported the initial deployment in addition to institution-wide support through the rescue from danger organisational redesign. CCRT response rates, ED wait times and SCU admission rates were continuously monitored to assess the effects of ER-STOP.

\section{Analytical approach}

After confirming that the control and intervention groups were similar in number, severity of illness and demographics, process and balancing measure data were tested for normality and then compared using t-tests or $\chi^{2}$ analysis, as appropriate, with two-tailed significance $(p<0.05)$. Control chart analysis ${ }^{9}$ using a standardised rule-based assessment for process and quality control evaluated the before-after change in monthly unexpected CCRT response using Microsoft Excel.

\section{Patient and public involvement}

The organisational rescue from danger redesign was prompted by a series of critical and severe adverse patient events related to deterioration within 24 hours of admission from the ED. The organisational response to such adverse events involves the transparent involvement of patients and families in the development of ER-STOP process. The quality improvement action plan was shared throughout development with select patient representatives and their families with their invaluable feedback incorporated into the final action plan. The organisation, as well as one of the author's, committed to the patients and families involved that these improvement measures would be implemented, measured and sustained using the expertise of hospital staff.

Given the large number of patients who are admitted annually from the ED, a multifaceted approach is necessary to ensure dissemination of study results to participants. The approach includes highlighting study results in the ED with posters and local waiting room TV promotional clips. Additionally, results were disseminated to patient relations and quality committees (both of which have patient membership), inclusion in the annual Quality and Safety fair, social media dissemination and inclusion in committee newsletters.

\section{RESULTS}

\section{Population measures}

There was no significant difference between the control and intervention groups in medical-surgical inpatient ward admission volume or severity of illness as measured by standardised national database resource intensity ${ }^{8}$ (table 1). This is reflected in the similarity of the top 10 national case-mix diagnostic groups ${ }^{10}$ for ward and medical short-stay unit (MSSU) admissions, inpatient length of stay and mortality (tables 2, 3 and 4).

\section{Process measures}

During the intervention period, there were 1748 admissions to a SCU, of which 93 admissions (5.32\%) were likely influenced by ER-STOP. Furthermore, the monthly proportion of pauses occurring in SCU admissions was consistent over the 12-month period (mean first 6 months 
Table 1 Medical-surgical inpatient ward admission volume and severity of illness

\begin{tabular}{|c|c|c|c|}
\hline & $\begin{array}{l}\text { Control } \\
\text { (Jan-Dec 2015) }\end{array}$ & $\begin{array}{l}\text { ER-STOP } \\
\text { (Jan-Dec 2016) }\end{array}$ & $P$ value \\
\hline Admissions ( $n)$ & 6069 & 6029 & \\
\hline
\end{tabular}

ER-STOP, Emergency Room Safer Transfer of Patients.

following deployment: $5.27 \pm 0.52 \%$, mean second 6 months following deployment: $5.38 \% \pm 0.46 \%, \mathrm{p}=0.7065$ ) with no evidence of a ramp-up effect.

The monthly rate of inpatient admissions with MEWS greater than or equal to 3 was also monitored following ER-STOP deployment to assess the impact on inpatient ward admission characteristics. These results (figure 2) show that the MSSU has the greatest monthly rate of admissions with MEWS greater than or equal to 3. Additionally, the data show a trend of increasing admissions to the MSSU with MEWS greater than or equal to 3 from January 2016 to December 2016. Criteria used to determine ER-STOP influence on patient admission are outlined in online supplementary figure 1 . Nursing team staffing and model of care did not change over the 2-year period of the study.

\section{Outcome measures}

Following the deployment of ER-STOP, the risk of an unexpected CCRT response following ED to adult medical and surgical ward admission was significantly decreased (OR 4.1, 95\% CI 2.17 to 7.77). Additionally, the monthly rate of unexpected CCRT responses decreased from 3.8 to $1.0(\mathrm{p}=0.001)$. These results are illustrated in figure 3. The u-control chart (figure 3) illustrates a time-series analysis of unexpected CCRT responses. This analysis shows a stable process with no significant variability in unexpected CCRT response rates before and after ER-STOP deployment. The distribution of hospital-wide unscheduled (new) to scheduled (follow-up consults+ICU discharge follow-up) CCRT responses was unchanged between the control and intervention periods $(\mathrm{p}=0.9185) \quad($ see table 5$)$.

Patient demographics and outcomes of unexpected CCRT responses within 24 hours of admission before and after ER-STOP are shown in table 6. Potentially high-risk ward admission prompting CCRT monitoring rather than emergent deployment occurred in eight patients (High dependency unit transfers $=3$, in-hospital mortality $=4$ ).

\begin{tabular}{|c|c|c|c|}
\hline Characteristic & 2015 & 2016 & $P$ value \\
\hline Admissions (n) & 6069 & 6029 & \\
\hline Gender (\% F/M) & $54.7 / 45.2$ & $53.2 / 46.7$ & 0.09 \\
\hline \multicolumn{4}{|l|}{ Age (years) } \\
\hline Minimum & 18 & 18 & 0.79 \\
\hline Mean & 66.7 & 66.8 & \\
\hline Median & 70 & 70 & \\
\hline $\begin{array}{l}\text { Maximum } \\
\text { SD }\end{array}$ & $\begin{array}{l}110 \\
19.6\end{array}$ & $\begin{array}{l}106 \\
19.7\end{array}$ & \\
\hline \multicolumn{4}{|l|}{ Length of stay (days) } \\
\hline Minimum & 1 & 1 & 0.82 \\
\hline Mean & 6.63 & 6.67 & \\
\hline Median & 4 & 4 & \\
\hline $\begin{array}{l}\text { Maximum } \\
\text { SD }\end{array}$ & $\begin{array}{l}171 \\
9.17\end{array}$ & $\begin{array}{l}139 \\
9.30\end{array}$ & \\
\hline Mortality (n) & 276 & 254 & 0.37 \\
\hline Mortality rate per 1000 patient days & 6.54 & 6.02 & 0.34 \\
\hline
\end{tabular}


Table 3 Top 10 case mix groups (CMGs) of inpatient admissions from emergency department

\begin{tabular}{|c|c|c|c|c|c|}
\hline \multicolumn{3}{|c|}{1 January 2015 to 31 December 2015} & \multicolumn{3}{|c|}{1 January 2016 to 31 December 2016} \\
\hline CMG & CMG description & Cases (n) & CMG & CMG description & Cases (n) \\
\hline 139 & $\begin{array}{l}\text { Chronic obstructive pulmonary } \\
\text { disease }\end{array}$ & 357 & 139 & Chronic obstructive pulmonary disease & 390 \\
\hline 810 & Palliative care & 175 & 671 & Organic mental disorder & 168 \\
\hline 138 & Viral/unspecified pneumonia & 156 & 810 & Palliative care & 165 \\
\hline 196 & $\begin{array}{l}\text { Heart failure without coronary } \\
\text { angiogram }\end{array}$ & 125 & 196 & Heart failure without coronary angiogram & 133 \\
\hline 671 & Organic mental disorder & 125 & 249 & Non-severe enteritis & 130 \\
\hline 026 & $\begin{array}{l}\text { Ischaemic event of central nervous } \\
\text { system }\end{array}$ & 123 & 026 & $\begin{array}{l}\text { Ischaemic event of central nervous } \\
\text { system }\end{array}$ & 120 \\
\hline
\end{tabular}

Unexpected ward mortality with full code resuscitation status remained unchanged before and after ER-STOP deployment $\left(\mathrm{n}_{2015}=13, \mathrm{n}_{2016}=16\right)$.

\section{Balancing measures}

Monthly mean ED wait times before and after ER-STOP remained unchanged. These data are summarised in table 7. The monthly average time from ED registration to admission before and after ER-STOP was 7.8 and 7.9 hours, respectively $(\mathrm{p}=0.39)$. Additionally, the monthly average time from ED admission to inpatient ward was 5.7 and 5.5 hours before and after deployment $(\mathrm{p}=0.192)$. Cardiac care unit admission rates before and after ER-STOP deployment remained unchanged at $9.51 \%$ and $9.60 \%$, respectively $(\mathrm{p}=0.198)$. ICU admission rates also remain unchanged in the control and intervention period with $3.84 \%$ admission rate prior to ER-STOP, and $4.61 \%$ following $(\mathrm{p}=0.06)$.

\section{DISCUSSION}

The results indicate that ER-STOP decreased unanticipated patient deterioration as measured by unexpected CCRT responses within 24 hours of admission from ED to a general adult ward by improving the matching of patient care needs to hospital unit location without significant increases in overall ED wait times or ED to SCU admissions. Unexpected CCRT responses were used as a surrogate index of patient safety wherein a safe admission would minimise the risk of patient deterioration and CCRT responses. An unexpected CCRT response

\begin{tabular}{|c|c|c|c|c|c|}
\hline \multicolumn{3}{|c|}{1 January 2015 to 31 December 2015} & \multicolumn{3}{|c|}{1 January 2016 to 31 December 2016} \\
\hline CMG & CMG description & Cases (n) & CMG & CMG description & Cases (n) \\
\hline 810 & Palliative care & 101 & 138 & Viral/unspecified pneumonia & 93 \\
\hline 487 & Lower urinary tract infection & 88 & 810 & Palliative care & 83 \\
\hline 671 & Organic mental disorder & 85 & 196 & $\begin{array}{l}\text { Heart failure without coronary } \\
\text { angiogram }\end{array}$ & 74 \\
\hline 196 & $\begin{array}{l}\text { Heart failure without coronary } \\
\text { angiogram }\end{array}$ & 81 & 670 & Dementia & 70 \\
\hline 477 & Renal failure & 67 & 026 & $\begin{array}{l}\text { Ischaemic event of central nervous } \\
\text { system }\end{array}$ & 54 \\
\hline
\end{tabular}


Time Series Plot of MSSU, A3, B3, F3, H7

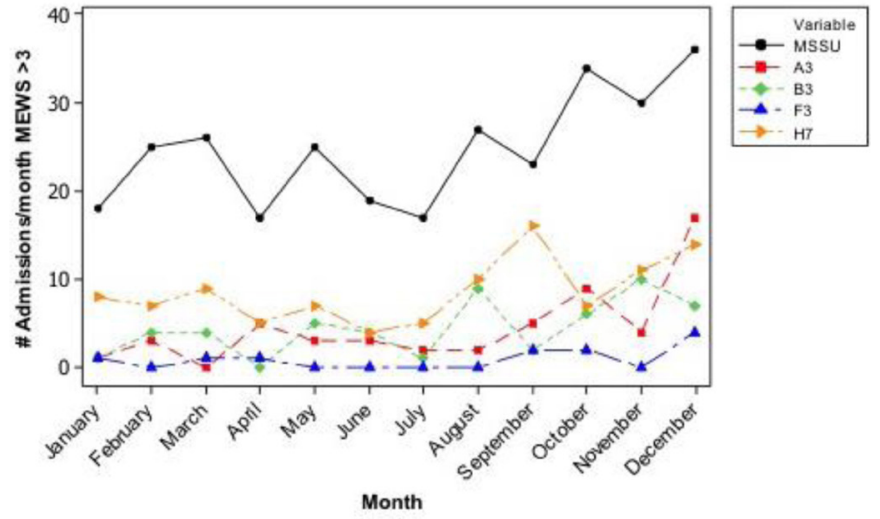

Figure 2 Monthly totals of unit-specific admissions with Modified Early Warning Score (MEWS) greater than or equal to 3. Black: medical short-stay unit (MSSU) admissions MEWS $\geq 3$; red: A3 general medical ward admissions MEWS $\geq 3$; green: B3 general medical ward admissions MEWS $\geq 3$; blue: F3 general medical ward admissions MEWS $\geq 3$; yellow: $\mathrm{H} 7$ general medical ward admissions MEWS $\geq 3$

can occur when patient care needs exceed ward capacity, clinical status deteriorates with increased risk of adverse outcomes including emergent SCU transfer and mortality, or with misalignment of patient/family goals of treatment. Audit of the transfer of accountability form revealed that ED staff use of ER-STOP redirected ED admissions from a general ward to SCUs and a consequential decrease in unexpected CCRT responses. Although ER-STOP reduced all CCRT responses within 24 hours of ED admission, total yearly CCRT responses were likely unchanged due to provincially mandated CCRT responses on ICU

Unexpected CCRT Responses within 24 hours of ED Admission Before and After Deployment of ER-STOP

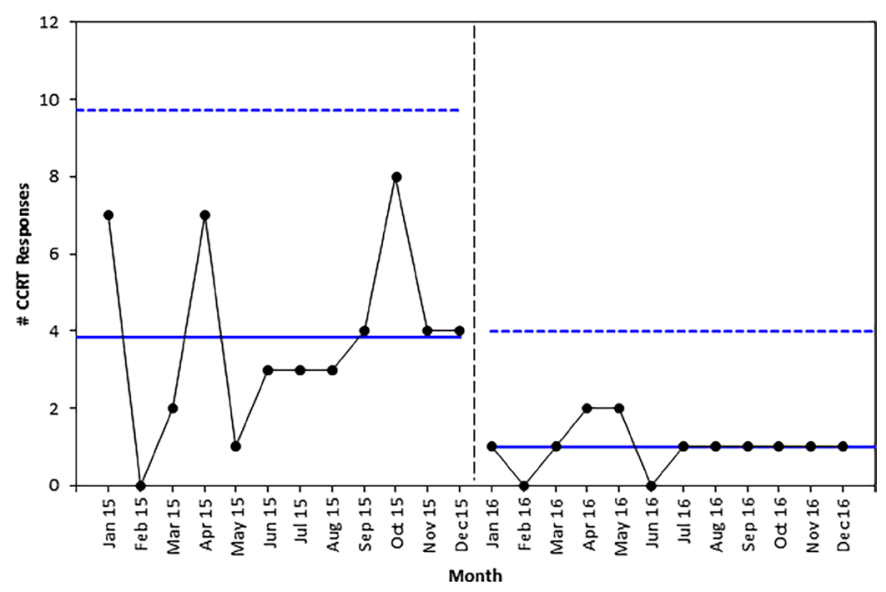

Figure 3 Control chart showing unexpected critical care response team (CCRT) responses within 24 hours of admission before (January 2015 to December 2015) and after (January 2016 to December 2016) deployment of Emergency Room Safer Transfer of Patients (ER-STOP). Blue line: mean monthly average of CCRT responses (before: 3.8, after: 1.0). Blue dashed line shows the upper control limit (before: 9.7, after: 3.83). ED, emergency department.
Table 5 Comparison of critical care response team responses

\begin{tabular}{lllll}
\hline & New & Follow-up & $\begin{array}{l}\text { ICU } \\
\text { discharge } \\
\text { follow-up }\end{array}$ & Total \\
\hline $\begin{array}{l}2015 \\
\text { consults }\end{array}$ & 513 & 517 & 910 & 1940 \\
consults & & & & \\
$\begin{array}{l}2016 \\
\text { (ER-STOP) }\end{array}$ & 541 & 554 & 945 & 2040 \\
\hline
\end{tabular}

ER-STOP, Emergency Room Safer Transfer of Patients; ICU, intensive care unit.

discharge and the standard practice of assuring clinical stability for 24 hours before CCRT sign-off. Interestingly, the distribution of scheduled and unscheduled CCRT responses in addition to mortality associated with full resuscitation status did not change during the study period suggesting more appropriate matching of patient need at the ward level. Overall SCU admission rates did not significantly increase, which was consistent with more appropriate use of SCUs by reducing early poor outcome admissions from general wards within 24 hours of admission. ER-STOP also led to implementation of non-SCU advanced directives.

In addition, with the deployment of ER-STOP, we observed a change in the function of the MSSU adjacent to the ED. The original purpose of this unit was to improve the efficiency of the inpatient admission process by providing care for admissions of under 36 hours' duration and accelerating diagnostic overnight investigations for inpatient ward admissions. Over the course of the ER-STOP deployment, the ward has transitioned into a close observation unit. Quantitative support for this change is the proportionally higher rate of monthly admissions to the MSSU with MEWS greater than 3. This observation is also supported qualitatively by interviews with MSSU staff, supervisors and the chief nursing executive. A change in the functionality of the MSSU ward may have also contributed to the success of ER-STOP as it provides an additional admission option for unstable patients requiring closer observation than is provided on a general ward.

The low prevalence of measured ward deterioration within 24 hours of ED admission limits the predictive effectiveness of the checklist component of the intervention, even with a high sensitivity and specificity. Previous work $^{7}$ and our local development process suggests a single MEWS would not have a high predictive test performance in this situation. Although our study was not designed to assess the predictive ability of the checklist components, the large number of patients admitted with MEWS greater than 3 (figure 2) suggests this component may have functioned more in a screening role with low positive predictive value. Therefore, the beneficial effect of ER-STOP's three-component intervention appears disproportionately greater than the modest predictive value of the quantitative checklist components. This might reflect the 
Table 6 Unexpected CCRT response patient demographics and outcomes following response

\begin{tabular}{|c|c|c|c|}
\hline \multicolumn{2}{|l|}{ Control } & \multicolumn{2}{|l|}{ ER-STOP } \\
\hline Average age (years) & 69.29 & Average age (years) & 63.08 \\
\hline $\mathrm{N}$ & 46 & $\mathrm{~N}$ & 12 \\
\hline Women & 18 & Women & 5 \\
\hline Transfer to high-capacity care unit & $14(30.4 \%)$ & Transfer to high-capacity care unit & $4(33 \%)$ \\
\hline Death & $13(28 \%)$ & Death & $4(33 \%)$ \\
\hline Discharge from ward & $33(72 \%)$ & Discharge from ward & $8(67 \%)$ \\
\hline
\end{tabular}

CCRT, critical care response team; ER-STOP, Emergency Room Safer Transfer of Patients.

enhanced ability of nurses to predict in-hospital mortality in comparison with physicians. ${ }^{11}$ However, it is more likely due to increased situational awareness and empowering a culture of patient safety. Situational awareness was cultivated in part by providing an explicit management plan to follow based on subjective staff nurse concern, wherein nurses were encouraged to consult with the most responsible physician and express concern regarding potential risk of patient deterioration.

Paramount to an empowered culture of patient safety has been the top-down hospital-wide rescue from danger redesign. This created a necessary organisational infrastructure required to educate and empower staff to use hospital-wide interventions and promote a culture of patient safety. In addition, ER-STOP was designed and validated within the local context. This ensured that the components of the project adequately served Michael Garron Hospital's (MGH) patient population, and the interventions were well integrated into existing systems to maximise their success.

In order to improve the function of ER-STOP, patient deterioration characteristics should be hardwired into the EHR at admission. As MGH ED shifts from a hybrid system to complete EHR, ER-STOP will be integrated into this system. As such, there will be standardised documentation, ability to flag patients for specific concerns and prompts for the admitting staff based on MEWS. The hardwiring of the ER-STOP during the admissions process should also increase staff adherence and ensure that all high-risk patients are reassessed to ensure patient need matches ward resources. In addition, the success of ER-STOP should be shared with ED staff in order to encourage them to continue using the checklist, purposeful pause and scripted management responses during patient admissions.

The limitations to this study are related to the single site deployment as the specific components of the checklist may not be applicable to other hospital populations without local adaptation, unmeasured changes in the severity of illness of the ED patient population, the lack of blinding of the intervention and the before-after design without a concurrent control group. However, the ED patient volumes in our hospital have consistently risen by approximately $3 \%$ annually over the period of the study with unchanged distribution of ED triage presentation scores suggesting an increasing burden of illness. Likewise, the investigators were not involved in the admission or unit allocation decisions. Although there is no evidence to suggest that scheduled CCRT responses prompted by ER-STOP on the day after ED admission substituted for unscheduled CCRT responses, we cannot exclude an indirect augmentation of ward care capacity by the brief presence of the CCRT on the ward.

\section{CONCLUSION}

In conclusion, deployment of a simple three-component intervention at ED admission improved patient safety outcomes by reducing high-risk unanticipated deterioration within 24 hours of admission. Additionally, balancing and process measures reveal no increase in special unit admission rate or $\mathrm{ED}$ wait times with wide uptake of this simple low-cost tool by ED staff. Although a formal economic analysis was not part of this study, no additional budgetary resources were allocated to this intervention and high-cost special care use was not significantly increased.

\begin{tabular}{llll}
\hline \multicolumn{1}{l}{ Table 7} & ED wait times before and after deployment of ER-STOP & & P value \\
\hline & Control & ER-STOP & 7.9 (SD 0.243) \\
$\begin{array}{l}\text { Monthly average time (mean hours) from ED } \\
\text { registration to admission }\end{array}$ & 7.8 (SD 0.273) & 0.39 \\
$\begin{array}{l}\text { Monthly average time (mean hours) from ED } \\
\text { admission to inpatient ward }\end{array}$ & 5.7 (SD 1.549) & 5.5 (SD 1.369) & 0.192
\end{tabular}

ED, emergency department; ER-STOP, Emergency Room Safer Transfer of Patients. 
The local development of ER-STOP allowed contextual adaptation to overcome limitations previously identified in early warning scores. The success of ER-STOP is likely related to increased situational awareness among ED staff. Local development and deployment is easily replicable in other settings using standardised audit analysis of deterioration events. Future process improvements associated with EHR integration, in contrast to the current hybrid paper model, will likely improve adherence and may improve patient outcomes

Furthermore, the organisational redesign has allowed for both bottom-up and top-down support of patient safety initiatives. Setting a hospital-wide goal of decreasing deterioration events allows for redesign of institutional processes and support culminating in sustainable patient safety initiatives.

\section{Author affiliations}

${ }^{1}$ Department of Medicine, Royal College of Surgeons in Ireland, Dublin, Ireland

${ }^{2}$ Operational Excellence and Sustainability, Michael Garron Hospital, Toronto,

Ontario, Canada

${ }^{3}$ Emergency Medicine, Michael Garron Hospital, Toronto, Ontario, Canada

${ }^{4}$ Department of Medicine, Division of Respirology, University of Toronto and Michael Garron Hospital, Michael Garron Hospital, Toronto, Ontario, Canada

Acknowledgements Dr James Yi for identifying the locally predictive components (MEWS \& Foley catheter) of the ER-STOP checklist as part of his family medicine academic residency project in 2014. We would also like to acknowledge Stephen Jerzak for his expertise in control chart design.

Contributors SN: Data analysis. Manuscript author. Attended and presented at BMJ IHI Forum. FDC: Ensured sustainability of ER-STOP. Provided valuable processbased feedback with respect to ER-STOP. Editing of manuscript. JS: ED staff nurse who assisted in the deployment process. Provided leadership for ED staff with respect to ER-STOP uptake. Frontline view on ER-STOP in practice which provided valuable feedback regarding the initiative. Editing of manuscript. IMF: Responsible for Outreach from Danger operational redesign. Provided top-down support for hospital-wide quality improvement initiatives. Development, integration and deployment of ER-STOP. Monitoring of intervention while performing on-call duties as attending physician. Editing and authorship of manuscript.

Funding The project was implemented and analysed using internal organisational resources without external funding.

Competing interests None declared.
Patient consent for publication Not required.

Ethics approval Reviewed and approved by the Research Ethics Board of the Michael Garron Hospital.

Provenance and peer review Not commissioned; externally peer reviewed.

Data sharing statement Extra data can be accessed via the Dryad data repository at http://datadryad.org/ with the doi:10.5061/dryad.v8kc47p.

Open access This is an open access article distributed in accordance with the Creative Commons Attribution Non Commercial (CC BY-NC 4.0) license, which permits others to distribute, remix, adapt, build upon this work non-commercially, and license their derivative works on different terms, provided the original work is properly cited, appropriate credit is given, any changes made indicated, and the use is non-commercial. See: http://creativecommons.org/licenses/by-nc/4.0/.

\section{REFERENCES}

1. Delgado MK, Liu V, Pines JM, et al. Risk factors for unplanned transfer to intensive care within 24 hours of admission from the emergency department in an integrated healthcare system. $J$ Hosp Med 2013;8:13-19.

2. Morgan RJ, Williams F, Wright MM. An early warning scoring system for detecting developing critical illness. Clin Intensive Care 1997;8:100.

3. Smith ME, Chiovaro JC, O'Neil M, et al. Early warning system scores for clinical deterioration in hospitalized patients: a systematic review. Ann Am Thorac Soc 2014;11:1454-65.

4. Alam N, Hobbelink EL, van Tienhoven AJ, et al. The impact of the use of the Early Warning Score (EWS) on patient outcomes: a systematic review. Resuscitation 2014;85:587-94.

5. Ko HC, Turner TJ, Finnigan MA. Systematic review of safety checklists for use by medical care teams in acute hospital settingslimited evidence of effectiveness. BMC Health Serv Res 2011;11:211.

6. Horwitz LI, Meredith T, Schuur JD, et al. Dropping the baton: a qualitative analysis of failures during the transition from emergency department to inpatient care. Ann Emerg Med 2009;53:701-10.

7. Churpek MM, Yuen TC, Winslow C, et al. Multicenter development and validation of a risk stratification tool for ward patients. Am J Respir Crit Care Med 2014;190:649-55.

8. Canadian Institute for Health Information Resource Indicators. DAD Resource intensity weights and expected length of stay. https://www. cihi.ca/en/resource-indicators-dad-resource-intensity-weights-andexpected-length-of-stay (accessed 25 Nov 2017).

9. Carey RG, Robert C. Lloyd measuring quality improvement in healthcare a guide to statistical process control measurements: American Society of Quality Quality Press, 2001.

10. Canadian Institute for Health Information. $\mathrm{CMG}+$. https://www.cihi. ca/en/cmg (accessed 25 Nov 2017).

11. Detsky ME, Harhay MO, Bayard DF, et al. Discriminative accuracy of physician and nurse predictions for survival and functional outcomes 6 months after an ICU admission. JAMA 2017;317:2187-95. 